Portland State University

PDXScholar

Electrical and Computer Engineering Faculty

Publications and Presentations

$5-1-1980$

\title{
Polarization characteristics of dye-laser amplifiers II. Isotropic molecular distributions
}

Lee W. Casperson

Portland State University

Kendall C. Reyzer

Follow this and additional works at: https://pdxscholar.library.pdx.edu/ece_fac

Part of the Electrical and Computer Engineering Commons

Let us know how access to this document benefits you.

\section{Citation Details}

Casperson, L. W., Reyzer, K. C. (1980). Polarization characteristics of dye-laser amplifiers II. Isotropic molecular distributions. Journal of Applied Physics, 51 (12), 6083-6090.

This Article is brought to you for free and open access. It has been accepted for inclusion in Electrical and Computer Engineering Faculty Publications and Presentations by an authorized administrator of PDXScholar. Please contact us if we can make this document more accessible: pdxscholar@pdx.edu. 


\title{
Polarization characteristics of dye-laser amplifiers II. Isotropic molecular distributions
}

\author{
Kendall C. Reyzer and Lee W. Casperson \\ School of Engineering and Applied Science, University of California, Los Angeles, California 90024
}

(Received 15 April 1980; accepted for publication 20 May 1980)

\begin{abstract}
In a previous study a general semiclassical formalism has been developed for lasers with arbitrary orientational distributions of the active molecules. Here that formalism is applied to the important case of an isotropic distribution of molecules, and this limit is especially relevant to liquid dye lasers. Extensive analytical and numerical results are presented for the polarization properties of such dye lasers. It is found that in general the polarization states of the pump and signal fields are coupled together and both change with distance in a laser amplifier.
\end{abstract}

PACS numbers: $42.55 . \mathrm{Mv}, 42.10 . \mathrm{Nh}$

\section{INTRODUCTION}

The gain of many laser systems depends strongly on the polarization states and directions of propagation of the optical signals. Dye lasers provide important examples of lasers with such anisotropic gain. In spite of this importance, however, there has been relatively little work done on the polarization properties of such lasers. As noted by Lempicki, none of the previous treatments had included the effects of gain saturation. ${ }^{1}$ We have recently discussed this situation and developed a semiclassical model for treating the polarization characteristics of dye-laser amplifiers. ${ }^{2}$ Of special interest in our previous study were those situations in which the active dipole moments are all parallel. In such cases the analysis is relatively simple and several analytic solutions can be obtained. It is clear, however, that a unidirectional molecular distribution is not the normal state of affairs in most practical dye lasers. Often when the dye molecules are suspended in liquids or plastics it is more reasonable to assume that the molecular distribution will be isotropic. The purpose of the present study is to apply the semiclassical formalism to dyelaser media having isotropic orientational distributions of the active dipoles. Most commercial dye lasers use a liquid host medium, and the results obtained here have implications for laser efficiency.

The analytical development in this work is based directly on the general formalism developed in our previous study. ${ }^{2}$ For compactness results obtained in Part I are referenced directly but with the equation numbers preceded by the number 1 . The isotropic oscillator concept is discussed briefly in Sec. II, and the general development for isotropic molecular distributions is presented in Sec. III. Most cases of interest have to be studied numerically, and several examples are considered in Sec. IV. Among the consequences of this study it is shown that the polarization of a propagating signal tends to rotate with distance toward the polarization orientation of the pump field. Somewhat less expected is the fact that with strong saturation by the signal the polarization of the pump tends to rotate away from the polarization of the signal. Results of this type have important implications for practical laser designs and several applications are considered in Sec. $\mathrm{V}$.

\section{THE ISOTROPIC OSCILLATOR}

Our main interest in the present study concerns molecules which in the absence of pump and stimulating fields have isotropic distributions of dipole moment orientations. This situation would correspond to most ordinary dye lasers, where the active molecules are suspended with random orientations in a liquid or solid medium. ${ }^{3}$ For an isotropic molecular distribution the function $n(\theta, \phi)$ is simply

$$
n(\theta, \phi)=N / 4 \pi \text {. }
$$

With this expression for the molecular distribution, the gain coefficients from Eqs. (I-44) are given by

$$
\begin{aligned}
& g_{x}(z)=\frac{N \sigma_{s}\left(v_{s}\right)}{4 \pi} \int_{\Omega} D_{s}(\theta, \phi, z) \cos ^{2} \theta d \Omega, \\
& g_{x y}(z)=\frac{N \sigma_{s}\left(v_{s}\right)}{4 \pi} \int_{\Omega} D_{s}(\theta, \phi, z) \cos \theta \sin \theta \cos \phi d \Omega, \\
& g_{y}(z)=\frac{N \sigma_{s}\left(v_{s}\right)}{4 \pi} \int_{\Omega} D_{s}(\theta, \phi, z) \sin ^{2} \theta \cos ^{2} \phi d \Omega .
\end{aligned}
$$

The simplest possible model for a laser transition can be termed the isotropic oscillator model. This model can be understood as requiring that the dipole moment of the laser transition is always exactly parallel to the signal polarization. With this assumption the interaction between the field and the dipoles is always a maximum. While the isotropic oscillator model is implicit in most discussions of stimulated emission, it is usually not rigorously valid. In an atomic system, for example, this model would require that the laser states must either be representable by spherically symmetric wavefunctions or else they must be imagined to align instantaneously with the signal field. In spite of these limitations the model is useful because of its simplicity, and more realistic models are discussed in the following sections.

In terms of the polarizability, the isotropic oscillator model assumes that the tensor is diagonal with all elements equal. Thus the induced moment is in the direction of the electric field, and the probability of exciting the oscillator is proportional to the amplitude squared of the field. Using this model for both the pump and signal fields, the population differences in Eq. (I-29) can be simplified since the terms 
involving $\left|\mathscr{E}_{p} \cdot \hat{e}_{p}\right|^{2}$ and $\left|\mathscr{E}_{s} \cdot \hat{e}_{s}\right|^{2}$ can be written

$$
\begin{aligned}
& \left|\mathscr{E}_{p} \cdot \hat{e}_{p}\right|^{2}=E_{p}^{2}, \\
& \left|\mathscr{E}_{s} \cdot \hat{e}_{s}\right|^{2}=E_{s}^{2},
\end{aligned}
$$

where $E_{p}$ and $E_{s}$ are the real amplitudes of the pump and stimulating electric fields. In this case, the gain coefficients from Eqs. (2) become (neglecting pump depletion)

$$
\begin{aligned}
& g_{x}(z)=N \sigma_{s}\left(v_{s 0}\right) \Phi_{p}^{2} /\left[1+\Phi_{p}^{2}+\Phi_{s}^{2}(z)\right], \\
& g_{x y}(z)=0, \\
& g_{y}(z)=N \sigma_{s}\left(v_{s 0}\right) \Phi_{p}^{2} /\left[1+\Phi_{p}^{2}+\Phi_{s}^{2}(z)\right] .
\end{aligned}
$$

Since $g_{x}$ and $g_{y}$ are equal, the gain can be defined as $g(z)$ and can be written

$$
g(z)=g_{0} /\left[1+s I_{s}(z)\right],
$$

where the unsaturated gain $g_{0}$ is given by

$$
g_{0}=N \sigma_{s}\left(v_{s 0}\right) \Phi_{p}^{2} /\left[1+\Phi_{p}^{2}\right]
$$

and the saturation parameter is

$$
s=\left[\tau_{3} \sigma_{s}\left(v_{\mathrm{so}}\right) / h v_{\mathrm{so}}\right] /\left[1 /\left(1+\Phi_{p}^{2}\right)\right] .
$$

It can be seen that the unsaturated gain from Eq. (6) is three times smaller than that found in Eq. (I-66). This is because the gain for the isotropic oscillator model is spherically symmetric while in the unidirectional situation the gain is present in the $x$ direction only. The unsaturated gain distribution from Eq. (I-60) becomes in this case

$$
g_{0}\left(\psi_{s}\right)=N \sigma_{s} \frac{\Phi_{p x}^{2}}{1+\Phi_{p x}^{2}}\left(\cos ^{2} \psi_{s}+\sin ^{2} \psi_{s}\right)
$$

which is the equation of a circle so that the gain distribution is uniform for any value of the pump field.

The unsaturated field and polarization angle equations from Eqs. (I-56) and (I-58) become

$$
\begin{aligned}
& E_{s}=E_{s 0} \exp \left[\frac{1}{2}\left(g_{0}-\gamma_{s}\right) z\right], \\
& \tan \psi_{s}=\tan \psi_{s 0},
\end{aligned}
$$

where $E_{x 0}$ is the initial field amplitude and $\psi_{s 0}$ is the initial polarization angle. Equation $(9 \mathrm{a})$ is the usual expression given for an unsaturated laser amplifier while Eq. (9b) means that the polarization direction remains fixed. When saturation is important, the field equations can be written in terms of the total intensity $I_{s}$ and using the gain expression in Eq. (5) become

$$
\frac{d I_{s}}{d z}=\frac{g_{0} I_{s}}{1+s I_{s}}-\gamma_{s} I_{s} .
$$

But this again is a standard laser amplifier equation.

\section{GENERAL ISOTROPIC DISTRIBUTION}

The rigorous solution to the general isotropic molecular distribution case can be obtained by substituting Eqs. (I-32) into Eqs. (2). Then each gain coefficient $g_{x}, g_{x y}$, and $g_{y}$ contains three terms, each of which involves an integral over $(\theta, \phi)$. Several of these integrals are the same so the gain terms can be simplified to

$$
g_{x}=N \sigma_{s}\left(\Phi_{p x}^{2} R_{1}+2 \Phi_{p x} \Phi_{p y} \cos \delta_{p} R_{2}+\Phi_{p y}^{2} R_{3}\right),
$$

$$
\begin{aligned}
& g_{x y}=N \sigma_{s}\left(\Phi_{p x}^{2} R_{2}+2 \Phi_{p x} \Phi_{p y} \cos \delta_{p} R_{3}+\Phi_{p y}^{2} R_{4}\right), \\
& g_{y}=N \sigma_{s}\left(\Phi_{p x}^{2} R_{3}+2 \Phi_{p x} \Phi_{p y} \cos \delta_{p} R_{4}+\Phi_{p y}^{2} R_{5}\right),
\end{aligned}
$$

where the $R_{i}$ are given by

$$
\begin{aligned}
& R_{1}(z)=\frac{3}{4 \pi} \int_{\Omega} \frac{\cos ^{4} \theta d \Omega}{1+f_{p}(\theta, \phi, z)+f_{s}(\theta, \phi, z)}, \\
& R_{2}(z)=\frac{3}{4 \pi} \int_{\Omega} \frac{\cos ^{3} \theta \sin \theta \cos \phi d \Omega}{1+f_{p}(\theta, \phi, z)+f_{s}(\theta, \phi, z)}, \\
& R_{3}(z)=\frac{3}{4 \pi} \int_{\Omega} \frac{\cos ^{2} \theta \sin ^{2} \theta \cos ^{2} \phi d \Omega}{1+f_{p}(\theta, \phi, z)+f_{s}(\theta, \phi, z)}, \\
& R_{4}(z)=\frac{3}{4 \pi} \int_{\Omega} \frac{\cos \theta \sin ^{3} \theta \cos ^{3} \phi d \Omega}{1+f_{p}(\theta, \phi, z)+f_{s}(\theta, \phi, z)}, \\
& R_{5}(z)=\frac{3}{4 \pi} \int_{\Omega} \frac{\sin ^{4} \theta \cos ^{4} \phi d \Omega}{1+f_{p}(\theta, \phi, z)+f_{s}(\theta, \phi, z)},
\end{aligned}
$$

The integrals in Eqs. (12) can be simplified after some straightforward algebraic manipulations and integrations, and the details of these operations are presented in the appendix. The results are

$$
\begin{aligned}
& R_{1}(z)=3 \int_{0}^{1} u^{4} I_{1 \phi}(u, z) d u, \\
& R_{2}(z)=3 \int_{0}^{1} u^{3}\left(1-u^{2}\right)^{1 / 2} I_{2 \phi}(u, z) d u, \\
& R_{3}(z)=3 \int_{0}^{1} u^{2}\left(1-u^{2}\right) I_{3 \phi}(u, z) d u, \\
& R_{4}(z)=3 \int_{0}^{1} u\left(1-u^{2}\right)^{3 / 2} I_{4 \phi}(u, z) d u, \\
& R_{5}(z)=3 \int_{0}^{1}\left(1-u^{2}\right)^{2} I_{5 \phi}(u, z) d u,
\end{aligned}
$$

where

$$
\begin{aligned}
I_{1 \phi}(u, z)= & \frac{1}{\sqrt{2}} \frac{1}{\left\{(A-C)+\left[\left(A+C^{2}\right)-B^{2}\right]^{1 / 2}\right\}^{1 / 2}} \\
& \times\left(\frac{1}{(A+B+C)^{1 / 2}}+\frac{1}{(A-B+C)^{1 / 2}}\right),
\end{aligned}
$$

$$
\begin{aligned}
I_{2 \phi}(u, z)= & \frac{1}{\sqrt{2}} \frac{1}{\left\{(A-C)+\left[(A+C)^{2}-B^{2}\right]^{1 / 2}\right\}^{1 / 2}} \\
& \times\left(\frac{1}{(A+B+C)^{1 / 2}}-\frac{1}{(A-B+C)^{1 / 2}}\right),
\end{aligned}
$$

$I_{3 \phi}(u, z)=\left[1-A I_{1 \phi}-B I_{2 \phi}\right] C^{-1}$,

$I_{4 \phi}(u, z)=\left[-B+A B I_{1 \phi}+\left(B^{2}-A C\right) I_{2 \phi}\right] C^{-2}$,

$$
\begin{aligned}
I_{5 \phi}(u, z)= & {\left[\left(C^{2} / 2\right)+\left(B^{2}-A C\right)-A\left(B^{2}-A C\right)\right.} \\
& \left.\times I_{1 \phi}-B\left(B^{2}-2 A C\right) I_{2 \phi}\right] C^{-3},
\end{aligned}
$$

and

$$
\begin{aligned}
& A(u, z)=1+\left[\Phi_{p x}^{2}(z)+\Phi_{s x}^{2}(z)\right] u^{2}, \\
& B(u, z)=2\left[\Phi_{p x}(z) \Phi_{p y}(z) \cos \delta_{p}(z)\right.
\end{aligned}
$$




$$
\begin{aligned}
& \left.+\Phi_{s x}(z) \Phi_{s y}(z) \cos \delta_{s}(z)\right] u\left(1-u^{2}\right)^{1 / 2}, \\
C(u, z)= & {\left[\Phi_{p y}^{2}(z)+\Phi_{s y}^{2}(z)\right]\left(1-u^{2}\right) . }
\end{aligned}
$$

The gain coefficients in Eqs. (11) can be calculated for any value of the pump and stimulating fields after obtaining the results from Eqs. (13) by numerical integration.

The unsaturated gain coefficients from Eqs. (11) and (12) are given by

$$
\begin{aligned}
& g_{x 0}=\frac{3 N \sigma_{s}}{4 \pi} \Phi_{p x}^{2} \int_{\Omega} \frac{\cos ^{4} \theta d \Omega}{1+\Phi_{p x}^{2} \cos ^{2} \theta}, \\
& g_{x y 0}=\frac{3 N \sigma_{s}}{4 \pi} \Phi_{p x}^{2} \int_{\Omega} \frac{\cos ^{3} \theta \sin \theta \cos \phi d \Omega}{1+\Phi_{p x}^{2} \cos ^{2} \theta}, \\
& g_{y 0}=\frac{3 N \sigma_{s}}{4 \pi} \Phi_{p x}^{2} \int_{\Omega} \frac{\cos ^{2} \theta \sin ^{2} \theta \cos ^{2} \phi d \Omega}{1+\Phi_{p x}^{2} \cos ^{2} \theta},
\end{aligned}
$$

which become after the integrations ${ }^{4}$

$$
\begin{aligned}
g_{x 0}= & N \sigma_{s}\left[1-\frac{3}{\Phi_{p x}^{2}}\left(1-\frac{\tan ^{-1} \Phi_{p x}}{\Phi_{p x}}\right)\right], \\
g_{x y 0}= & 0 \\
g_{y 0}= & N \sigma_{s} \\
& \times\left[1+\frac{3}{2 \Phi_{p x}^{2}}\left(1-\frac{\left(\Phi_{p x}^{2}+1\right) \tan ^{-1} \Phi_{p x}}{\Phi_{p x}}\right)\right] .
\end{aligned}
$$

The unsaturated gain distribution for the isotropic molecular distribution is found by using Eqs. (17a) and (17c) in Eq. (I-60). Figure 1 is a plot of the dimensionless gain distribution for various values of the pump field. From the expressions for $g_{x 0}$ and $g_{y 0}$ given in Eqs. (17a) and (17c) it is seen that $g_{x 0}$ and $g_{y 0}$ both approach $N \sigma_{s}$ in the limit of infinite pump intensity and the unsaturated gain distribution becomes uniform. This is an expected result for as the pump intensity increases, the probability of exciting those dipoles

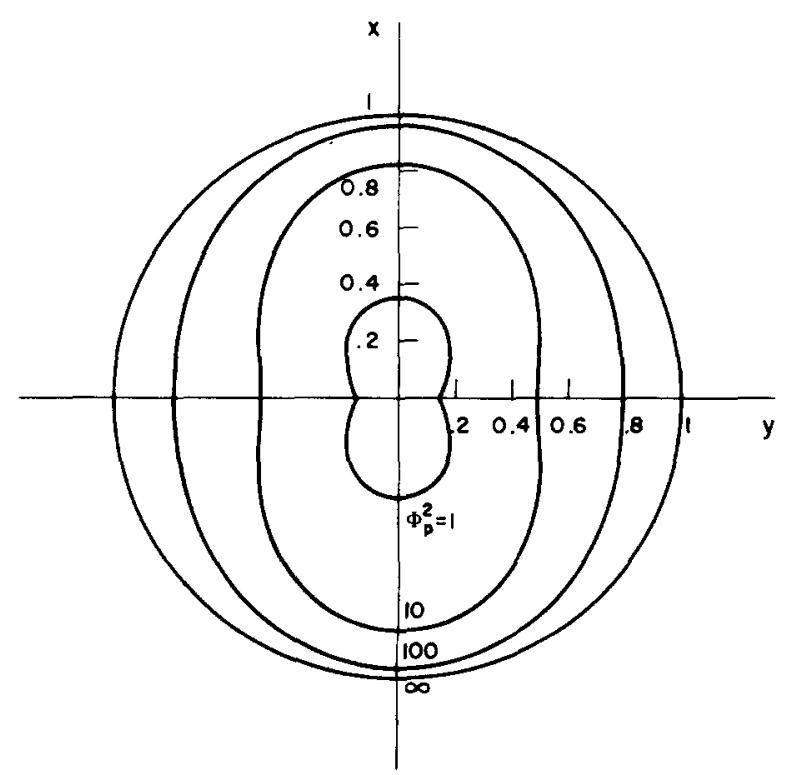

FIG. 1. Unsaturated gain distribution for an isotropic molecular ensemble excited by a linearly polarized pump. that are oriented close to the $y$ axis increases, thus increasing the gain in the $y$ direction. As the pump intensity approaches infinity, the gain distribution becomes uniform because all the available molecules are excited. The molecules that are oriented exactly perpendicular to the pump will not be excited, but their contribution to the total gain is negligible.

When saturation becomes important, the gain expressions become more complicated and require a numerical solution. There are two cases, however, when the gain expressions are integrable. If the stimulating field is initially polarized parallel to the pump field then from Eq. (I- 33b), $f_{s}(0)=\Phi_{s x}^{2}(0) \cos ^{2} \theta$, and the integration over $\phi$ in Eq. (2b) is zero by symmetry. Thus with $g_{x y}(0)$ equal to zero the $\mathrm{cw}$ gain terms in Eqs. (I-43a) and (I-43b) become

$$
\begin{aligned}
& \left.\frac{d E_{s x}}{d z}\right|_{z=0}=\frac{1}{2} g_{x}(0) E_{s x}(0), \\
& \left.\frac{d E_{s y}}{d z}\right|_{z=0}=0,
\end{aligned}
$$

and it is clear that $E_{s y}$ is zero for all $z$ and the stimulating field remains linearly polarized in the $x$ direction. Similarly if the stimulating field is initially polarized along the $y$ direction then

$$
\begin{aligned}
& \left.\frac{d E_{s x}}{d z}\right|_{z=0}=0, \\
& \left.\frac{d E_{s y}}{d z}\right|_{z=0}=\frac{\log }{2} g_{y}(0) E_{s y}(0),
\end{aligned}
$$

and the stimulating field remains linearly polarized in the $y$ direction.

From Eqs. (I-33a) and (I-40b), $\alpha_{x y}(0)$ is zero in the two cases discussed above and the derivatives in Eqs. (I-39a) and (I-39b) become

$$
\begin{aligned}
& \left.\frac{d E_{p x}}{d z}\right|_{z=0}=-\frac{1}{2} \alpha_{x}(0) E_{p x}(0), \\
& \left.\frac{d E_{p y}}{d z}\right|_{z=0}=0
\end{aligned}
$$

so the pump field remains linearly polarized along the $x$ direction.

With the above results, the gain coefficients from Eqs. (11) for the stimulating field polarized parallel and perpendicular to the pump field are given by ${ }^{5}$

$$
\begin{aligned}
g_{x_{x}}= & N \sigma_{s} \frac{\Phi_{p x}^{2}}{\Phi_{p x}^{2}+\Phi_{s x}^{2}}\left[1-\frac{3}{\left(\Phi_{p x}^{2}+\Phi_{s x}^{2}\right)}\right. \\
& \left.\times\left(1-\frac{\tan ^{-1}\left(\Phi_{p x}^{2}+\Phi_{s x}^{2}\right)^{1 / 2}}{\left(\Phi_{p x}^{2}+\Phi_{s x}^{2}\right)^{1 / 2}}\right)\right], \\
g_{x y_{1}}= & 0, \\
g_{y_{1}}= & N \sigma_{s} \frac{\Phi_{p x}^{2}}{\Phi_{p x}^{2}+\Phi_{s x}^{2}}\left[1+\frac{3}{2\left(\Phi_{p x}^{2}+\Phi_{s x}^{2}\right)}\right. \\
& \left.\times\left(1-\frac{\left(1+\Phi_{p x}^{2}+\Phi_{s x}^{2}\right) \tan ^{-1}\left(\Phi_{p x}^{2}+\Phi_{s x}^{2}\right)^{1 / 2}}{\left(\Phi_{p x}^{2}+\Phi_{s x}^{2}\right)^{1 / 2}}\right)\right],
\end{aligned}
$$


$g_{x_{1}}=\frac{N \sigma_{s}}{\left(\Phi_{p x}^{2}-\Phi_{s y}^{2}\right)^{2}}\left[f_{x_{1}}+f_{x_{1}} E(w, q)-f_{x_{s}} F(w, q)\right]$,

$g_{x y_{3}}=0$,

$g_{y_{i}}=\frac{N \sigma_{s} \Phi_{p x}^{2}}{\left(\Phi_{p x}^{2}-\Phi_{s y}^{2}\right)^{2} \Phi_{s y}^{2}}$ $\times\left[f_{y_{1}}-f_{y_{2}} E(w, q)+f_{y_{3}} F(w, q)\right]$,

where

$$
\begin{aligned}
& f_{x_{1}}=\Phi_{p x}^{2}\left(\Phi_{p x}^{2}-3\right)-\Phi_{s y}^{2}\left(3 \Phi_{p x}^{2}-1\right), \\
& f_{x_{2}}=2\left(1+\Phi_{s y}^{2}\right)^{1 / 2}\left[2 \Phi_{p x}^{2}+\Phi_{s y}^{2}\left(\Phi_{p x}^{2}-1\right)\right] / \Phi_{p x}, \\
& f_{x_{s}}=\left(1+\Phi_{s y}^{2}\right)^{1 / 2}\left(\Phi_{p x}^{2}-\Phi_{s y}^{2}\right) / \Phi_{p x}, \\
& f_{y_{1}}=\Phi_{s y}^{2}\left(2+\Phi_{p x}^{2}+\Phi_{s y}^{2}\right) \\
& f_{y_{2}}=\left(1+\Phi_{s y}^{2}\right)^{1 / 2}\left[\Phi_{p x}^{2}+\Phi_{s y}^{2}\left(2 \Phi_{p x}^{2}+1\right)\right] / \Phi_{p x}, \\
& f_{y_{3}}=\left(1+\Phi_{s y}^{2}\right)^{1 / 2}\left(\Phi_{p x}^{2}-\Phi_{s y}^{2}\right) / \Phi_{p x} .
\end{aligned}
$$

The functions $E(w, q)$ and $F(w, q)$ are incomplete elliptic integrals of the first and second kind respectively with parameters $w$ and $q$ given by

$$
\begin{aligned}
& w=\tan ^{-1} \Phi_{p x}, \\
& q=\frac{\Phi_{s y}}{\Phi_{p x}}\left(\frac{\Phi_{p x}^{2}+1}{\Phi_{s y}^{2}+1}\right)^{1 / 2} .
\end{aligned}
$$

Similar results can also be obtained for the absorption coefficients. These results are quite complicated and ultimately require numerical methods to solve for the field dependences.

\section{GENERAL SOLUTIONS}

The purpose of this section is to present numerical solutions for the important case of arbitrary pump and signal fields propagating in a laser medium having an isotropic distribution of the active dipoles. In order to simplify the numerical techniques that are necessary to solve Eqs. (I-39) and (I-43) and to minimize the number of variable parameters, the equations are first normalized. The absorption coefficients given in Eqs. (I-40) can be written in a form similar to the gain terms in Eqs. (11) and are proportional to $N \sigma_{p}$. Then using Eqs. (I-28), (I-36), and (I-37) the absorption and stimulated cross sections can be written as

$$
\begin{gathered}
\sigma_{p}\left(v_{p}\right)=\frac{\sigma_{p 0}\left(v_{p} / v_{p 0}\right)}{1+K_{p}^{2}}, \\
\sigma_{s}\left(v_{s}\right)=\frac{\sigma_{s 0}\left(v_{s} / v_{s 0}\right)}{1+K_{s}^{2}},
\end{gathered}
$$

where $\sigma_{p 0}$ and $\sigma_{s 0}$ are the absorption and stimulated emission cross sections at line center, and the ratios $v_{p} / v_{p 0}$ and $v_{s} / v_{s 0}$ may be taken to be unity. The maxima of the absorption and emission cross sections are approximately equal in many dyes so the line center cross section is defined as

$$
\sigma_{0}=\sigma_{p 0}=\sigma_{s)} \text {. }
$$

The gain and absorption coefficients then become

$$
\begin{aligned}
& g_{x}(z)=N \sigma_{0} g_{x}^{\prime}(z) /\left(1+K_{s}^{2}\right)=N \sigma_{0} g_{x}^{\prime \prime}(z), \\
& g_{x y}(z)=N \sigma_{0} g_{x y}^{\prime}(z) /\left(1+K_{s}^{2}\right)=N \sigma_{0} g_{x y}^{\prime \prime}(z),
\end{aligned}
$$

$$
\begin{aligned}
& g_{y}(z)=N \sigma_{0} g_{y}^{\prime}(z) /\left(1+K_{s}^{2}\right)=N \sigma_{0} g_{y}^{\prime \prime}(z), \\
& \alpha_{x}(z)=N \sigma_{0} \alpha_{x}^{\prime}(z) /\left(1+K_{p}^{2}\right)=N \sigma_{0} \alpha_{x}^{\prime \prime}(z), \\
& \alpha_{x y}(z)=N \sigma_{0} \alpha_{x y}^{\prime}(z) /\left(1+K_{p}^{2}\right)=N \sigma_{0} \alpha_{x y}^{\prime \prime}(z), \\
& \alpha_{y}(z)=N \sigma_{0} \alpha_{y}^{\prime}(z) /\left(1+K_{p}^{2}\right)=N \sigma_{0} \alpha_{y}^{\prime \prime}(z) .
\end{aligned}
$$

Since the dimensionless field amplitudes from Eq. (I-34) are directly proportional to the electric field and since the gain and absorption coefficients are directly proportional to $N \bar{\sigma}_{0}$ from Eqs. (28) and (29), the normalized amplitude and phase equations can be written

$$
\begin{aligned}
& \frac{d \Phi_{p x}}{d \xi}=-\frac{\alpha_{x}^{\prime \prime}}{2} \Phi_{p x}-\frac{\alpha_{x y}^{\prime \prime}}{2} \Phi_{p y}\left[\cos \delta_{p}+K_{p} \sin \delta_{p}\right], \\
& \frac{d \Phi_{p y}}{d \xi}=-\frac{\alpha_{y}^{\prime \prime}}{2} \Phi_{p y}-\frac{\alpha_{x y}^{\prime \prime}}{2} \Phi_{p x}\left[\cos \delta_{p}-K_{p} \sin \delta_{p}\right],
\end{aligned}
$$

$$
\begin{aligned}
\frac{d \delta_{p}}{d \xi}= & \frac{\alpha_{x y}^{\prime \prime}}{2}\left(K_{p} \frac{\left(\Phi_{p x}^{2}-\Phi_{p y}^{2}\right)}{\Phi_{p x} \Phi_{p y}} \cos \delta_{p}\right. \\
& \left.+\frac{\left(\Phi_{p x}^{2}+\Phi_{p y}^{2}\right)}{\Phi_{p x} \Phi_{p y}} \sin \delta_{p}\right)-K_{p} \frac{\left(\alpha_{x}^{\prime \prime}-\alpha_{y}^{\prime \prime}\right)}{2},
\end{aligned}
$$$$
\frac{d \Phi_{s x}}{d \xi}=\frac{g_{x}^{\prime \prime}}{2} \Phi_{s x}+\frac{g_{x y}^{\prime \prime}}{2} \Phi_{s y}\left[\cos \delta_{s}+K_{s} \sin \delta_{s}\right],
$$$$
\frac{d \Phi_{s y}}{d \xi}=\frac{g_{y}^{\prime \prime}}{2} \Phi_{s y}+\frac{g_{x y}^{\prime \prime}}{2} \Phi_{s x}\left[\cos \delta_{s}-K_{s} \sin \delta_{s}\right],
$$$$
\frac{d \delta_{s}}{d \xi}=-\frac{g_{x y}^{\prime \prime}}{2}\left(K_{s} \frac{\left(\Phi_{s x}^{2}-\Phi_{s y}^{2}\right)}{\Phi_{s x} \Phi_{s y}} \cos \delta_{s}\right.
$$

$$
\left.+\frac{\left(\Phi_{s x}^{2}+\Phi_{s y}^{2}\right)}{\Phi_{s x} \Phi_{s y}} \sin \delta_{s}\right)+\frac{K_{s}\left(g_{x}^{\prime \prime}-g_{y}^{\prime \prime}\right)}{2}
$$

where $\xi$ is a dimensionless distance parameter defined by

$$
\xi=N \sigma_{0} z \text {. }
$$

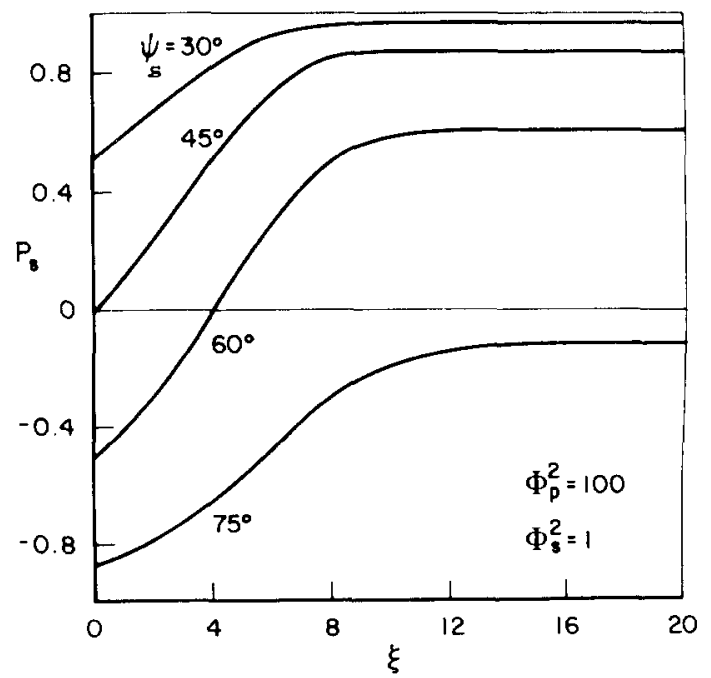

FIG. 2. Degree of polarization of the stimulating field as a function of the normalized distance into the gain medium for various values of the initial direction of the stimulating field. 
The time derivatives have been dropped and the loss term is assumed to be much smaller than the absorption and gain terms and is ignored. Equations (30) and (31) are coupled first-order nonlinear differential equations that can be solved by numerical integration.

The solutions to Eqs. (30) and (31) depend on the initial conditions of the pump and stimulating fields and on the offline-center parameters $K_{p}$ and $K_{s}$. A dimensionless pump field intensity and a dimensionless stimulating field intensity can be defined as

$$
\begin{aligned}
& \Phi_{p}^{2}=\Phi_{p x}^{2}+\Phi_{p y}^{2}, \\
& \Phi_{s}^{2}=\Phi_{s x}^{2}+\Phi_{s y}^{2} .
\end{aligned}
$$

The initial pump field is assumed linearly polarized in the $x$ direction and the initial value of $\Phi_{p}^{2}$ is taken to be 100 . This corresponds to a pumping level of approximately 10 $\mathrm{MW} / \mathrm{cm}^{2}$, which is a reasonable value for pumping with unfocused doubled Nd:YAG lasers. It is also assumed that $K_{p}$ and $K_{s}$ are zero, but this condition will be relaxed later.

If the stimulating field is polarized at some angle $\psi_{s}$ to the $x$ axis then the $x$ and $y$ components of the stimulating field are coupled. This coupling occurs by the cross saturation of the $x$ and $y$ gain and also through the $g_{x y}^{\prime \prime}$ term. Assume first however that the stimulating field is infinitesimal and the gain is unsaturated. The initial values of the derivatives from Eqs. (31) become in this case

$$
\begin{aligned}
& \left.\frac{d \Phi_{s x}}{d \xi}\right|_{\xi=0}=\frac{1}{2} g_{x 0}^{\prime \prime}(0) \Phi_{s x}(0), \\
& \left.\frac{d \Phi_{s y}}{d \xi}\right|_{\xi=0}=\frac{1}{2} g_{y 0}^{\prime \prime}(0) \Phi_{s y}(0),
\end{aligned}
$$

so both field components must increase. But the $x$ component of the field will see a larger gain than the $y$ component due to the gain anisotropy and as a consequence the stimulating field must rotate toward the $x$ axis. As saturation becomes important the growth of the fields decreases as does

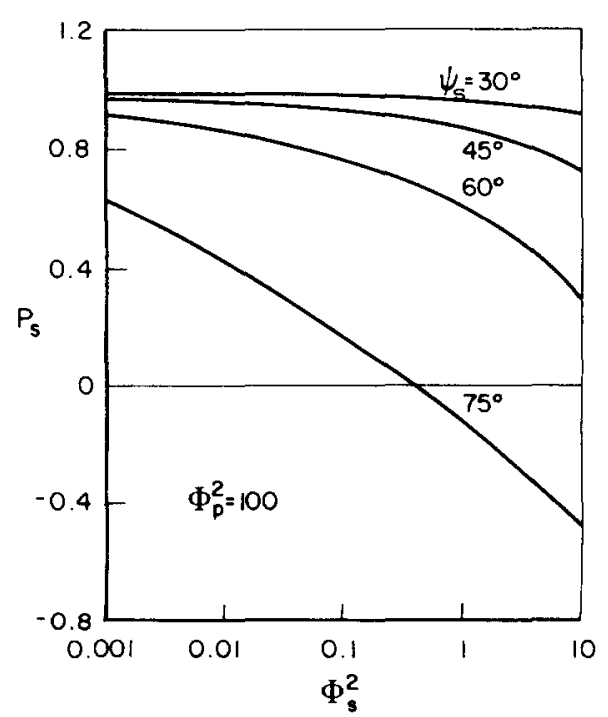

FIG. 3. Maximum value of the degree of polarization of the stimulating field as a function of the input intensity for various values of the direction of the stimulating field.

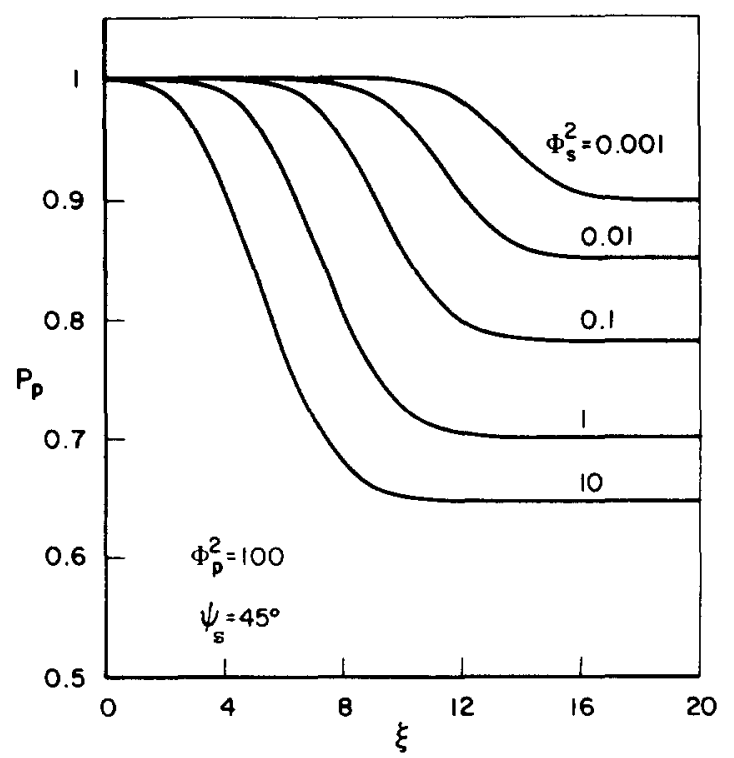

FIG. 4. Degree of polarization of the pump field as a function of the normalized distance into the gain medium for various values of the initial intensity of the stimulating field.

the amount of rotation due to the decreased gain and to the $g_{x y}^{\prime \prime}$ coupling. Effectively the $g_{x y}^{\prime \prime}$ term acts as a loss since $g_{x y}^{\prime \prime}$ is negative. Physically this represents an excited dipole that is stimulated by one component of the field and thus is unavailable for stimulation by the orthogonal field component. field as

Defining a degree of polarization for the stimulating

$$
P_{s}=\left(\Phi_{s x}^{2}-\Phi_{s y}^{2}\right) /\left(\Phi_{s x}^{2}+\Phi_{s y}^{2}\right)
$$

a rotation of the stimulating field toward the $x$ axis means that $\boldsymbol{P}_{s}$ approaches a value of one. This can be seen in the numerical results presented in Figs. 2 and 3. Figure 2 shows the increase in $P_{s}$ with increasing distance into the gain medium while Fig. 3 shows the effects of saturation on the

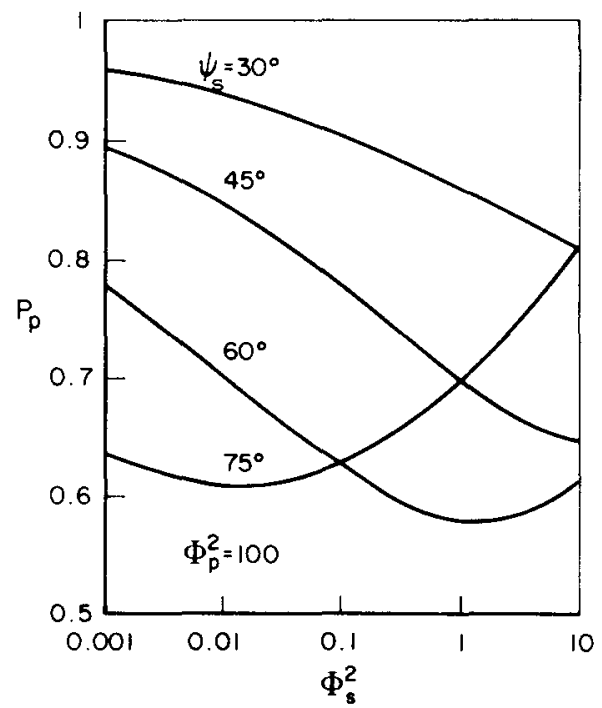

FIG. 5. Minimum value of the degree of polarization of the pump field as a function of the initial intensity for various values of the direction of the stimulating field. 
amount of rotation the stimulating field will experience. It is evident from these figures that with large input signals less signal rotation is possible.

When the stimulating field is polarized at some angle $\psi_{s}$ to the $x$ axis, the pump field also is affected. This occurs because $\alpha_{x y}^{\prime \prime}$ is nonzero. The initial values of the derivatives in Eqs. (30) become

$$
\begin{aligned}
& \left.\frac{d \Phi_{p x}}{d \xi}\right|_{\xi=0}=-\frac{1}{2} \alpha_{x}^{\prime \prime}(0) \Phi_{p x}(0), \\
& \left.\frac{d \Phi_{p y}}{d \xi}\right|_{\xi=0}=-\frac{1}{2} \alpha_{x y}^{\prime \prime}(0) \Phi_{p x}(0), \\
& \left.\frac{d \delta_{p}}{d \xi}\right|_{\xi=0}=0,
\end{aligned}
$$

and so the $y$ component of the pump field grows and the polarization of the pump field rotates away from the $x$ direction with a sense that is determined by the rotation of the stimulating field. The rotation (clockwise or counterclockwise) is the same for both the pump and stimulating fields.

Defining a degree of polarization for the pump field as

$$
P_{p}=\left(\Phi_{p x}^{2}-\Phi_{p y}^{2}\right) /\left(\Phi_{p x}^{2}+\Phi_{p y}^{2}\right)
$$

the effects of the stimulating field on the direction of the pump field can be seen in Figs. 4 and 5. For the pump field, a rotation away from the $x$ direction means that $P_{p}$ will decrease from its initial value of one. Figure 4 shows the decrease in $P_{p}$ with increasing distance into the gain medium while Fig. 5 shows the minimum value that $P_{p}$ will attain depending on the initial direction and intensity of the stimulating field. It can be seen that the pump rotation is very dependent on the saturation of the gain. For the stimulating field oriented close to the $x$ axis the rotation increases with increasing intensity of the stimulating field while for orientations near the $y$ axis the amount of rotation will first increase then decrease as a function of the stimulating field intensity. This is caused by an increase in the $y$ component of the ab-

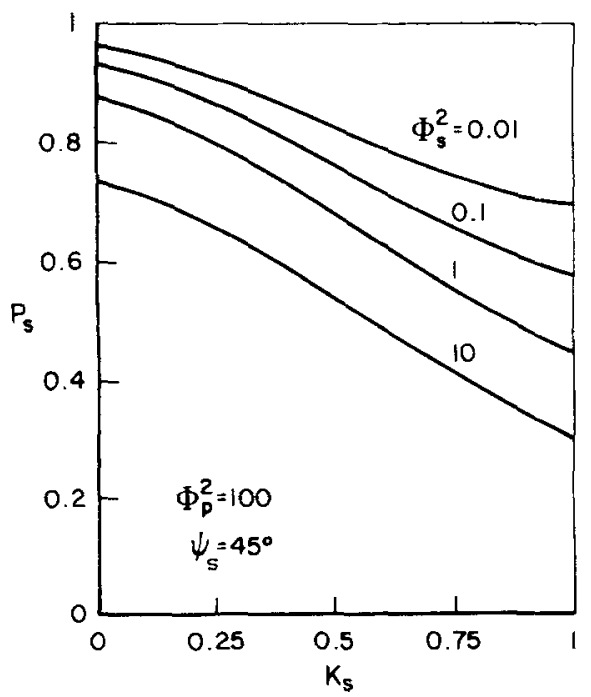

FIG. 6. Variation in the maximum degree of polarization of the stimulating field as a function of the frequency offset from line center for various values of the signal intensity.

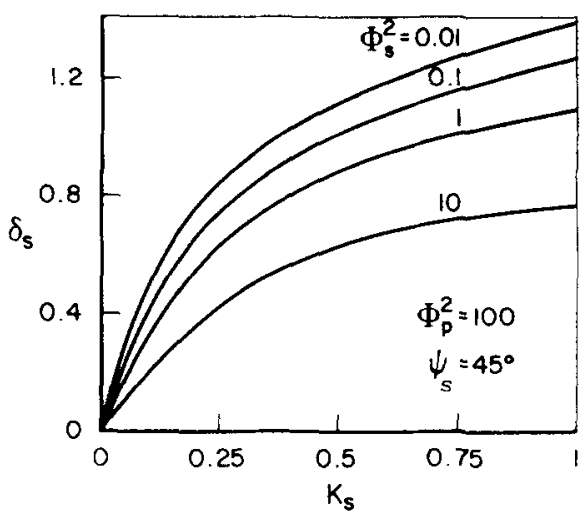

FIG. 7. Maximum phase difference between the $x$ and $y$ components of the stimulating field as a functioin of the frequency offset from line center for various values of the stimulating field intensity.

sorption coefficient due to the strong saturation of the $y$ component of the gain.

If the frequency of the stimulating field is not at line center, the parameter $K_{s}$ is no longer zero. From Eq. (31c) the derivative term at $\xi=0$ is nonzero and the phase difference between the $x$ and $y$ components of the stimulating field must change. In this situation the medium becomes birefringent and the stimulating field becomes elliptically polarized. As the frequency of the stimulating field gets farther away from line center, $K_{s}$ gets larger as seen in Eq. (I-46) and correspondingly $\sigma_{s}$ gets smaller from Eq. (26b). In this case the gain coefficients decrease and the degree of polarization of the stimulating field clearly does not change as much as at line center. This is seen in Fig. 6. There is still a rotation toward the $x$ axis but in this situation it is the direction of the major axis of the elliptically polarized stimulating field that rotates toward the $x$ axis. The phase difference between the $x$

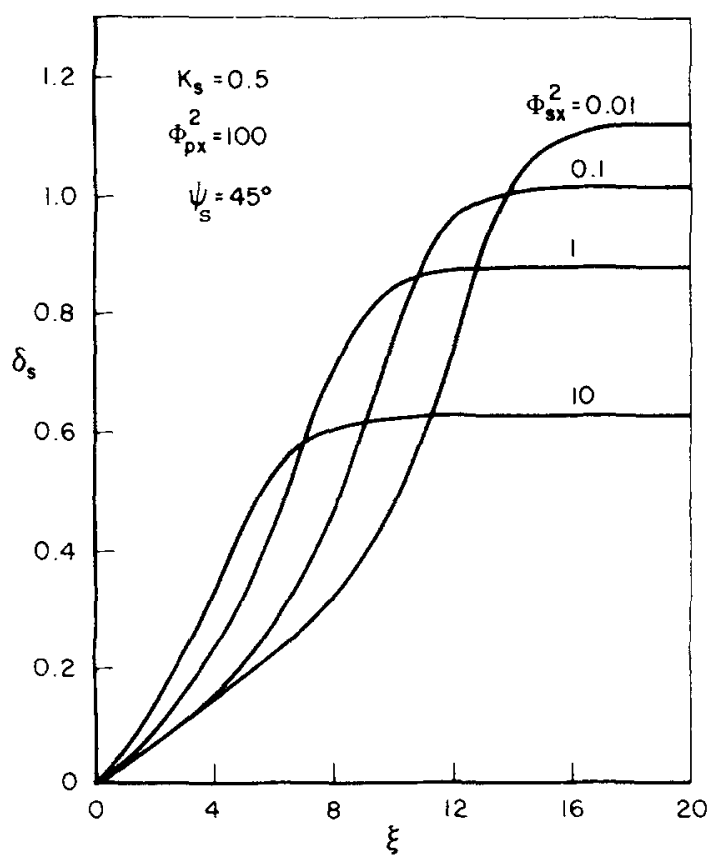

FIG. 8. Phase difference as a function of the normalized distance into the gain medium for various values of the input intensity of the stimulating field. 
and $y$ components of the stimulating field increases rapidly as $K_{s}$ changes. This is seen in Fig. 7 which also shows that increased intensity in the initial stimulating field reduces the phase difference since the gain terms become saturated. Finally, Fig. 8 shows how the phase difference increases with increasing distance into the gain medium and also shows the effects of saturation. The case when $K_{p}$ is nonzero is very similar to the above case in that the pumping efficiency decreases due to a decrease in the absorption coefficients from Eq. (29), and thus the gain also decreases. The pump field becomes elliptically polarized as the derivative in Eq. (30c) is nonzero.

\section{DISCUSSION}

In this paper new analytical and numerical results have been presented dealing with the polarization of the pump and signal fields in a laser-pumped light amplifier. It has been shown that the polarization state of both the pump and signal fields can rotate as they propagate along the amplifying medium. The rotations may be due to the anisotropic distribution of gain and loss that occur with a linearly polarized pump laser field. These results suggest that using a polarized laser as a pump source is an inherently inefficient process when the initial molecular distribution is isotropic, and such effects can be important in dye and far infrared gas lasers. However, if the initial distribution of molecules is modified in some way to favor a particular direction, then the pumping process becomes more efficient.

There are several possible techniques that could be used to provide a preferred molecular orientation such as electric field orientation of a permanent dipole moment, shear flow orientation of long molecular chains, stretching of a plastic film that contains the molecules, or orientation in a liquid crystal. ${ }^{6}$ The use of liquid crystal solvents has been the subject of several recent papers and appears to be a practical method of producing a strong repeated orientation of the dye molecules with a reasonably small applied field. ${ }^{7,8}$

The ability to orient the molecules will not only improve efficiency, but it can also provide a means for modulating the gain and absorption of an active medium. Thus one may consider the case of a pump field polarized along the $x$ axis while the alignment of the molecules is switched between the $x$ and $y$ axes. In this case the pump absorption will change from a maximum to a minimum, and the effect resembles an electro-optical switch. An advantage is that the modulation voltage would be very low if a liquid crystal could be used. Obviously, the response time of such a device would depend on the speed with which the liquid crystal can orient the molecules. In a similar manner, the gain maximum can be switched between the $x$ and $y$ axes so that a linearly polarized signal field can be modulated. This approach would be preferable to the absorption method for applications where both gain and modulation are required.

Another possible modulation technique is opto-optic modulation where a strong signal of one polarization is modulated by a weak signal at the orthogonal polarization due to cross saturation of the gain. ${ }^{9}$ As seen previously, the $x$ and $y$ components of a stimulating field are coupled through the $g_{x y}$ term in addition to the saturation of the gain by both components. If a stimulating field is polarized along the $y$ axis when the gain maximum occurs along $x$, then the introduction of a small control signal polarized along $x$ can modulate the incremental gain seen by the stimulating field.

\section{APPENDIX}

The integrals in Eqs. (12) can be simplified by making the substitution $U=\cos \theta$. The integrals then become

$$
\begin{aligned}
& R_{1}(z)=\frac{3}{2} \int_{-1}^{1} u^{4} \\
& \times\left[\frac{1}{2 \pi} \int_{0}^{2 \pi} \frac{d \phi}{A(u, z)+B(u, z) \cos \phi+C(u, z) \cos ^{2} \phi}\right] d u \\
& R_{2}(z)=\frac{3}{2} \int_{-1}^{1} u^{3}\left(1-u^{2}\right)^{1 / 2} \\
& \times\left[\frac{1}{2 \pi} \int_{0}^{2 \pi} \frac{\cos \phi d \phi}{A(u, z)+B(u, z) \cos \phi+C(u, z) \cos ^{2} \phi}\right] d u \\
& R_{3}(z)=\frac{3}{2} \int_{-1}^{1} u^{2}\left(1-u^{2}\right) \\
& \times\left[\frac{1}{2 \pi} \int_{0}^{2 \pi} \frac{\cos { }^{2} \phi d \phi}{A(u, z)+B(u, z) \cos \phi+C(u, z) \cos ^{2} \phi}\right] d u \\
& R_{4}(z)=\frac{3}{2} \int_{-1}^{1} u\left(1-u^{2}\right)^{3,2} \\
& \times\left[\frac{1}{2 \pi} \int_{0}^{2 \pi} \frac{\cos ^{3} \phi d \phi}{A(u, z)+B(u, z) \cos \phi+C(u, z) \cos ^{2} \phi}\right] d u \\
& R_{5}(z)=\frac{3}{2} \int_{-1}^{1}\left(1-u^{2}\right)^{2} \\
& \times\left[\frac{1}{2 \pi} \int_{0}^{2 \pi} \frac{\cos ^{4} \phi d \phi}{A(u, z)+B(u, z) \cos \phi+C(u, z) \cos ^{2} \phi}\right] d u
\end{aligned}
$$

where

$$
\begin{aligned}
A(u, z)= & 1+\left[\Phi_{p x}^{2}(z)+\Phi_{s x}^{2}(z)\right] u^{2} \\
B(u, z)= & 2\left[\Phi_{p x}(z) \Phi_{p y}(z) \cos \delta_{p}(z)\right. \\
& \left.+\Phi_{s x}(z) \Phi_{s y}(z) \cos \delta_{s}(z)\right] u\left(1-u^{2}\right)^{1 / 2} \\
C(u, z)= & {\left[\Phi_{p y}^{2}(z)+\Phi_{s y}^{2}(z)\right]\left(1-u^{2}\right) }
\end{aligned}
$$

Looking at the $\phi$ integrals in Eqs. (A 1) and defining them as $I_{1 \phi}$ through $I_{5 \phi}$, these integrals can be written after some simple algebraic manipulations as

$$
\begin{aligned}
I_{3 \phi}= & {\left[1-A I_{1 \phi}-B I_{2 \phi}\right] C^{-1}, } \\
I_{4 \phi}= & {\left.\left[-B+A B I_{1 \phi}+\left(B^{2}-A C\right) I_{2 \phi}\right] C^{-2}, \quad \text { (A 3b }\right) } \\
I_{5 \phi}= & {\left[\left(C^{2} / 2\right)+\left(B^{2}-A C\right)\right.} \\
& \left.-A\left(B^{2}-A C\right) I_{1 \phi}-B\left(B^{2}-2 A C\right) I_{2 \phi}\right] C^{-3} .
\end{aligned}
$$

The solution to Eqs. (A 3) requires completing the integration of $I_{1 \phi}$ and $I_{2 \phi}$. The integrals are written as

$I_{1 \phi}(u, z)$ 
$=\frac{1}{2 \pi} \int_{0}^{2 \pi} \frac{d \phi}{A(u, z)+B(u, z) \cos \phi+C(u, z) \cos ^{2} \phi}$,

(A4a)

$$
\begin{aligned}
& I_{2 \phi}(u, z) \\
& \quad=\frac{1}{2 \pi} \int_{0}^{2 \pi} \frac{\cos \phi d \phi}{A(u, z)+B(u, z) \cos \phi+C(u, z) \cos ^{2} \phi} .
\end{aligned}
$$

Separating the integrals into the two intervals $[0, \pi]$ and $[\pi, 2 \pi]$ and then transforming the integrals over $[\pi, 2 \pi]$ back to $[0, \pi]$ yields

$$
\begin{aligned}
& I_{1 \phi}=I_{1 \phi}^{+}+I_{1 \phi}^{-}, \\
& I_{2 \phi}=I_{2 \phi}^{+}-I_{2 \phi}^{-},
\end{aligned}
$$

where

$$
\begin{aligned}
& I_{1 \phi}^{+}=\frac{1}{2 \pi} \int_{0}^{\pi} \frac{d \phi}{A+B \cos \phi+C \cos ^{2} \phi}, \\
& I_{1 \phi}^{-}=\frac{1}{2 \pi} \int_{0}^{\pi} \frac{d \phi}{A-B \cos \phi+C \cos ^{2} \phi}, \\
& I_{2 \phi}^{+}=\frac{1}{2 \pi} \int_{0}^{\pi} \frac{\cos \phi d \phi}{A+B \cos \phi+C \cos ^{2} \phi}, \\
& I_{2 \phi}=\frac{1}{2 \pi} \int_{0}^{\pi} \frac{\cos \phi d \phi}{A-B \cos \phi+C \cos ^{2} \phi} .
\end{aligned}
$$

Making the substitution $v=\tan (\phi / 2)$, the integrals in Eqs. (A 6) can be written

$$
\begin{aligned}
& I_{1 \phi}^{+}=\frac{1}{2 \pi} \int_{0}^{\infty} \frac{2\left(1+v^{2}\right) d v}{a+2 b v^{2}+c v^{4}}, \\
& I_{1 \phi}=\frac{1}{2 \pi} \int_{0}^{\infty} \frac{2\left(1+v^{2}\right) d v}{c+2 b v^{2}+a v^{4}}, \\
& I_{2 \phi}^{+}=\frac{1}{2 \pi} \int_{0}^{\infty} \frac{2\left(1-v^{2}\right) d v}{a+2 b v^{2}+c v^{4}}, \\
& I_{2 \phi}^{-}=\frac{1}{2 \pi} \int_{0}^{\infty} \frac{2\left(1-v^{2}\right) d v}{c+2 b v^{2}+a v^{4}},
\end{aligned}
$$

where

$$
\begin{aligned}
& a=A+B+C, \\
& b=A-C, \\
& c=A-B+C .
\end{aligned}
$$

Two similar terms appear in each of the integrals in Eqs. (A 7) and the solutions for these terms are given by ${ }^{10}$

$$
\begin{aligned}
& \int_{0}^{\infty} \frac{d v}{\alpha+2 \beta v^{2}+\gamma v^{4}}=\frac{\pi}{2 \sqrt{ } 2} \frac{1}{\sqrt{ } \alpha\left[\beta+(\alpha \gamma)^{1 / 2}\right]^{1 / 2}} \\
& \int_{0}^{\infty} \frac{v^{2} d v}{\alpha+2 \beta v^{2}+\gamma v^{4}}=\frac{\pi}{2 \sqrt{ } 2} \frac{1}{\sqrt{ } \gamma\left[\beta+(\alpha \gamma)^{1 / 2}\right]^{1 / 2}}
\end{aligned}
$$

With these results, $I_{1 \phi}$ and $I_{2 \phi}$ become

$$
\begin{aligned}
I_{1 \phi}= & \frac{1}{\sqrt{ } 2} \frac{1}{\left[b+(a c)^{1 / 2}\right]^{1 / 2}}\left(\frac{1}{\sqrt{ } a}+\frac{1}{\sqrt{ } c}\right) \\
= & \frac{1}{\sqrt{ } 2} \frac{1}{\left\{(A-C)+\left[(A+C)^{2}-B^{2}\right]^{1 / 2}\right\}^{1 / 2}} \\
& \times\left(\frac{1}{(A+B+C)^{1 / 2}}+\frac{1}{(A-B+C)^{1 / 2}}\right), \\
I_{2 \phi}= & \frac{1}{\sqrt{ } 2} \frac{1}{\left[b+(a c)^{1 / 2}\right]^{1 / 2}}\left(\frac{1}{\sqrt{ } a}-\frac{1}{\sqrt{ } c}\right), \\
= & \frac{1}{\sqrt{ } 2} \frac{1}{\left\{(A-C)+\left[(A+C)^{2}-B^{2}\right]^{1 / 2}\right\}^{1 / 2}} \\
& \times\left(\frac{1}{(A+B+C)^{1 / 2}}-\frac{1}{(A-B+C)^{1 / 2}}\right) .
\end{aligned}
$$

From Eq. (A 2) it is seen that $A$ and $C$ are even functions of $u$ while $B$ is an odd function of $u$. It follows then from Eqs. (A 10) that $I_{1 \phi}$ is an even function of $u$ and $I_{2 \phi}$ is an odd function of $u$. Using the symmetry of $I_{1 \phi}$ and $I_{2 \phi}$, the symmetries of $I_{3 \phi}, I_{4 \phi}$, and $I_{5 \phi}$ are readily established from Eqs. (A 3). With these results, Eqs. (A 1) can be written as

$$
\begin{aligned}
& R_{1}(z)=3 \int_{0}^{1} u^{4} I_{1 \phi}(u, z) d u, \\
& R_{2}(z)=3 \int_{0}^{1} u^{3}\left(1-u^{2}\right)^{1 / 2} I_{2 \phi}(u, z) d u, \\
& R_{3}(z)=3 \int_{0}^{1} u^{2}\left(1-u^{2}\right) I_{3 \phi}(u, z) d u, \\
& R_{4}(z)=3 \int_{0}^{1} u\left(1-u^{2}\right)^{3 / 2} I_{4 \phi}(u, z) d u, \\
& R_{5}(z)=3 \int_{0}^{1}\left(1-u^{2}\right)^{2} I_{5 \phi}(u, z) d u .
\end{aligned}
$$

These results appear as Eqs. (13) in the text.

'A. Lempicki, Acta Phys. Pol. A 50, 179 (1976).

${ }^{2}$ K. C. Reyzer and L. W. Casperson, J. Appl. Phys. 51 (1980).

${ }^{3}$ J. Frenkel, Kinetic Theory of Liquids (Dover, New York, 1955)

${ }^{4}$ I. S. Gradshteyn and I. W. Ryzhik, Tables of Integrals, Series and Products (Academic, San Francisco, 1965), Eq. 2.562-2.

${ }^{5}$ Ref. 4, Eqs. 3.153-1 and 3.154-1.

${ }^{6}$ E. D. Baily and B. R. Jennings, Appl. Opt. 11, 527 (1972).

${ }^{7}$ M. Bertolotti, G. Sansoni, and F. Scudieri, Appl. Opt. 18, 528 (1979).

${ }^{8}$ I. P. Il-chishin, E. A. Tikhonov, M. T. Shpak, and A. A. Doroshkin, JETP Lett. 24, 303 (1976).

${ }^{9}$ R. W. Gray and L. W. Casperson, IEEE J. Quantum Electron. QE14, 893 (1978).

${ }^{10}$ Ref. 4, Eqs. 2.161-1 and 3.252-9. 